\title{
Triethanolamine: A Resourceful, Reusable, Eco-Friendly, Reaction Medium for Phase Transfer Catalyst - Free Synthesis of 1-(Arylsulfonyl)aryl/heterylmethanes
}

\author{
K. SRINIVAS ${ }^{1^{*}}$ and P.K. DUBEY ${ }^{2}$ \\ ${ }^{1}$ Department of Chemistry, GITAM Institute of Technology, \\ GITAM University, Rushikonda, Visakhapatnam (A.P.) - 530 045, India \\ ${ }^{2}$ Department of Chemistry, College of Engineering, Jawaharlal Nehru Technological \\ University, Hyderabad, Kukatpally, Hyderabad (A.P.) - 500 085, India \\ kummarisrinivas@gmail.com
}

Received 6 March 2013 / Accepted 30 April 2013

\begin{abstract}
Reaction of arylmethyl/heteryl methyl chloride (1) with aryl sulphinate sodium salt (2) yields the corresponding sulphone derivative promoted by triethanolamine at room temperature.
\end{abstract}

Keywords: Arylmethyl/heteryl methyl chloride, Aryl sulphinate sodium salt, Triethanol amine

\section{Introduction}

The aryl sulfones are having common structures in valuable molecules in fields such as pharmaceuticals, agrochemicals and polymer sciences ${ }^{1}$. In particular, their immense utilities in medicinal chemistry and their unique bioactivities have attracted considerable attention on their synthesis. For example, diaryl sulfones have been reported to inhibit HIV $-1^{2}$ reverse transcriptase and diphenyl sulfone ${ }^{3}$ is used as an intermediate for the synthesis of 4,4'-diamino-diphenyl sulfone (DAPSONE), which is effective for leprosy treatment ${ }^{4}$. The aryl sulfones can be prepared from the transition metal-catalyzed reactions using sulfonic acids or sulfonyl chloride ${ }^{5}$ but the pre-functionalizing such as metallisation or halogenations of arenas are required. A well-known process involving the formation of new C-S bond from aromatic $\mathrm{C}-\mathrm{H}$ bonds is the Friedel-Crafts (FC) sulfonylation of various arenes, especially electron-rich arenas ${ }^{6,7}$. Sulfonchlorides or sulfoanhydrides are general substrates employed in the FC sulfonylation.

It is well known that chiral amino alcohols, such as natural amino alcohols, carbohydrate- and camphor-derived amino alcohols and ferrocenyl amino alcohols, have been widely used as ligands $(\mathrm{O}, \mathrm{N}$-ligands) in, for example, the asymmetric alkynylation of aldehydes $^{8}$, the asymmetric addition of diethylzinc to aldehydes and imines ${ }^{9}$, the asymmetric ruthenium-catalysed transfer hydrogenation of ketones ${ }^{10}$, the enantioselective trimethylsilylcyanation of aldehydes ${ }^{11}$ and in enantioelective henry reactions ${ }^{12}$. Furthermore, 
Buchwald and co-workers have disclosed that trans-1,2-diaminocyclohexane serves as an excellent ligand ( $N, N$-ligand) in the copper-catalysed amidation of aryl halides and in the $N$-arylation of $N$-heterocycles (C,N bond coupling) ${ }^{13}$ and ethylene glycol as a ligand (O,O-ligand) in the copper-catalysed amination of aryl iodides (C-N bond coupling) ${ }^{14}$ in 2001 and 2002, respectively. Most recently, Gozalez-Bobes and Fu discovered that amino alcohols such as prolinol and trans-2-aminocyclohexanol are effective ligands (O,N-ligands) in the nickel-catalysed Suzuki reactions of unactivated alkyl halides, including secondary alkyl chlorides, with arylboronic acids (C-C bond coupling) ${ }^{15}$. However, triethanolamine exhibited lower activity in the Heck reaction of chlorobenzene with styrene in water using catalytic palladium on charcoal ${ }^{16}$.

Herein we report on triethanolamine, cheap and commercially available organic compound, an efficient and recyclable, phase transfer catalyst free and as reaction medium in synthesis of 1-(arylsulfonyl) aryl/heteryl methanes.

In continuation of our earlier work ${ }^{17-20}$ on synthesis of new heteryl derivatives with potential biological activity, we now wish to report the preparation of the title compounds under phase transfer catalyst (PTC)-free conditions i.e. without using any PTC, in the presence of triethanol amine (TEOA) as resourceful reaction medium at room temperature. The results of these studies are presented in this communication.

\section{Experimental}

Melting points are uncorrected and were determined in open capillary tubes in sulphuric acid bath. TLC was performed on silica gel-G and spotting was done using iodine or UV-light. IR spectra were recorded with Perkin-Elmer 1000 instrument in $\mathrm{KBr}$ phase. ${ }^{1} \mathrm{H}$ NMR was recorded on VARIAN 400MHz instrument and Mass spectra were recorded on Agilent-LCMS instrument. The following experimental procedures are reprehensive of the general procedures used to synthesize all compounds.

\section{General procedure for preparation of $\mathbf{3 a - j}$}

A mixture of 1 (10 mmol), $2(10.1 \mathrm{mmol})$ and triethanol amine were stirred at RT till the reaction was complete, as shown by TLC. After carrying out the reaction, the mixture was filtered and the filtrate extracted with diethyl ether [note: the solubility of Triethanolamine in diethyl ether is approx $1.2-1.4 \%$ at $\left.25^{\circ} \mathrm{C}\right]^{23}$. Triethanolamine was separated and washed successively with $\mathrm{Et}_{2} \mathrm{O}(2 \times 5 \mathrm{~mL})$ and hexane $(5 \mathrm{~mL})$ in order to remove adsorbed organic substrates. It can be reused directly without further purification. The physical and spectra data of the compounds 3a-i are as follows.

\section{2-(Toluene-4-sulfonylmethyl)-1H-benzimidazole (3a)}

M.P. $\left({ }^{\circ} \mathrm{C}\right): 201$ [Lit: $202^{21}$ ]; IR (KBr) cm ${ }^{-1}: 3000(\mathrm{NH}), 1302\left(\mathrm{SO}_{2}\right) ;{ }^{1} \mathrm{H}-\mathrm{NMR}\left(\mathrm{DMSO}_{6}\right): \delta$ $2.4\left(\mathrm{~s}, 3 \mathrm{H},-\mathrm{CH}_{3}\right), 4.95\left(\mathrm{~s}, 2 \mathrm{H},-\mathrm{CH}_{2}\right), 7.2-7.7(\mathrm{~m}, 8 \mathrm{H}, \mathrm{Ar}-\mathrm{H}), 12.6$ (bs, $1 \mathrm{H},-\mathrm{NH}, \mathrm{D}_{2} \mathrm{O}$ exchangeable); $\mathrm{M}^{+}+1$ : 287; Anal. Calcd. for $\left(\mathrm{C}_{15} \mathrm{H}_{14} \mathrm{~N}_{2} \mathrm{O}_{2} \mathrm{~S}\right)$ requires: $\mathrm{C}, 62.92 ; \mathrm{H}, 4.93 ; \mathrm{N}$, 9.78\%; Found: C, 62.86; H, 4.87; N, 9.74\%.

\section{2-Benzenesulfonylmethyl-1H-benzimidazole (3b)}

M.P. $\left({ }^{\circ} \mathrm{C}\right): 199-201$ [Lit: 198-200 ${ }^{21}$ ]; IR (KBr) cm ${ }^{-1}: 3426(\mathrm{NH}), 1324\left(\mathrm{SO}_{2}\right) ;{ }^{1} \mathrm{H}-\mathrm{NMR}$ $\left(\right.$ DMSO-d $\left._{6}\right): \delta 4.85\left(\mathrm{~s}, 2 \mathrm{H},-\mathrm{CH}_{2}\right), 7.15-7.80(\mathrm{~m}, 9 \mathrm{H}, \mathrm{Ar}-\mathrm{H}), 12.65$ (bs, $1 \mathrm{H},-\mathrm{NH}, \mathrm{D}_{2} \mathrm{O}$ exchange- able); $\mathrm{M}^{+}+1$ : 273 ; Anal. Calcd. for $\left(\mathrm{C}_{14} \mathrm{H}_{12} \mathrm{~N}_{2} \mathrm{O}_{2} \mathrm{~S}\right)$ requires: $\mathrm{C}, 61.75$; $\mathrm{H}, 4.44$; N, 10.29\%; Found: C, 61.70; H, 4.40; N, 10.26\%. 


\section{2-[1-(Toluene-4-sulfonyl)-ethyl]-1H-benzimidazole (3c)}

M.P. $\left({ }^{\circ} \mathrm{C}\right): 155$ [Lit: $154-56^{21}$ ]; IR (KBr) cm ${ }^{-1}: 3388(\mathrm{NH}), 1308\left(\mathrm{SO}_{2}\right) ;{ }^{1} \mathrm{H}-\mathrm{NMR}$ (DMSO$\left.\mathrm{d}_{6}\right): \delta 1.84\left(\mathrm{~d}, J=7.16 \mathrm{~Hz}, 3 \mathrm{H},-\mathrm{CH}-\mathrm{CH}_{3}\right), 2.36\left(\mathrm{~s}, 3 \mathrm{H},-\mathrm{C}_{6} \mathrm{H}_{4}-\mathrm{CH}_{3}-(p)\right), 4.68(\mathrm{q}, J=7.14 \mathrm{~Hz}$, $1 \mathrm{H},-\mathrm{CH}-\mathrm{CH}_{3}$ ), 7.2-7.7 (m, 8H, Ar-H), 10.3 (bs, $1 \mathrm{H},-\mathrm{NH}-, \mathrm{D}_{2} \mathrm{O}$ exchangeable); $\mathrm{M}^{+}+1: 301$; Anal. Calcd. for $\left(\mathrm{C}_{16} \mathrm{H}_{16} \mathrm{~N}_{2} \mathrm{O}_{2} \mathrm{~S}\right)$ requires: $\mathrm{C}, 63.98 ; \mathrm{H}, 5.37 ; \mathrm{N}, 9.33 \%$; Found: C, 63.94; $\mathrm{H}$, $5.35 ; \mathrm{N}, 9.30 \%$.

\section{2-(1-Benzenesulfonyl-ethyl)-1H-benzimdazole (3d)}

M.P. $\left({ }^{\circ} \mathrm{C}\right): 181$ [Lit: $180^{21}$ ]; IR $(\mathrm{KBr}) \mathrm{cm}^{-1}: 3000,1308\left(\mathrm{SO}_{2}\right) ;{ }^{1} \mathrm{H}-\mathrm{NMR}$ (DMSO-d $\left.\mathrm{d}_{6}\right): \delta 1.9$ (d, $J=7.16 \mathrm{~Hz}, 3 \mathrm{H},-\mathrm{CH}-\mathrm{CH}_{3}$ ), 4.7 (q, $\left.J=7.12 \mathrm{~Hz}, 1 \mathrm{H},-\mathrm{CH}_{-} \mathrm{CH}_{3}\right), 7.2-7.7$ (m, 9H, Ar-H), 10.3 (bs, $1 \mathrm{H},-\mathrm{NH}, \mathrm{D}_{2} \mathrm{O}$ exchangeable); $\mathrm{M}^{+}+1$ : 287; Anal. Calcd. for $\left(\mathrm{C}_{15} \mathrm{H}_{14} \mathrm{~N}_{2} \mathrm{O}_{2} \mathrm{~S}\right)$ requires: $\mathrm{C}$, 62.92; H, 4.93; N, 9.78\%; Found: C, 62.86; H, 4.91; N, 9.74\%.

\section{1-Methyl-2-(toluene-4-sulfonylmethyl)-1H-benzimidazole (3e)}

M.P. $\left({ }^{\circ} \mathrm{C}\right): 206 ; \mathrm{IR}(\mathrm{KBr}) \mathrm{cm}^{-1}: 1300\left(\mathrm{SO}_{2}\right) ;{ }^{1} \mathrm{H} \mathrm{NMR}\left(\mathrm{CDCl}_{3}\right): \delta 2.45$ (s, 3H, $\left.-\mathrm{CH}_{3}\right), 3.95$ (s, $\left.3 \mathrm{H},-\mathrm{NCH}_{3}\right), 4.75\left(\mathrm{~s}, 2 \mathrm{H},-\mathrm{CH}_{2}\right), 7.2-7.7(\mathrm{~m}, 8 \mathrm{H}, \mathrm{Ar}-\mathrm{H}) ; \mathrm{M}^{+}+1$ : 301 ; Anal. Calcd. for $\left(\mathrm{C}_{16} \mathrm{H}_{16} \mathrm{~N}_{2} \mathrm{O}_{2} \mathrm{~S}\right)$ requires: C, 63.98; H, 5.37; N, 9.33\%; Found: C, 63.94; H, 5.32; N, 9.28\%.

\section{2-Benzenesulfonylmethyl-1-methyl-1H-benzimidazole (3g)}

M.P. $\left({ }^{\circ} \mathrm{C}\right): 172 ; \mathrm{IR}(\mathrm{KBr}) \mathrm{cm}^{-1}: 1336\left(\mathrm{SO}_{2}\right) ;{ }^{1} \mathrm{H}$ NMR $\left(\mathrm{CDCl}_{3}\right): \delta 1.85(\mathrm{~d}, J=7.18 \mathrm{~Hz}, 3 \mathrm{H},-$ $\left.\mathrm{CH}_{2}-\mathrm{CH}_{3}\right), 2.40$ (s, 3H, - $\left.\mathrm{C}_{6} \mathrm{H}_{4}-\mathrm{CH}_{3}-(p)\right), 3.95$ (s, 3H, $\left.-\mathrm{NCH}_{3}\right), 4.70$ (q, J=7.0 Hz, 1H, -CH$\left.\mathrm{CH}_{3}\right)$, 7.2-7.7 (m, 8H, Ar-H); $\mathrm{M}^{+}+1$ : 315 ; Anal. Calcd. for $\left(\mathrm{C}_{17} \mathrm{H}_{18} \mathrm{~N}_{2} \mathrm{O}_{2} \mathrm{~S}\right)$ requires: $\mathrm{C}$, 64.94; H, 5.77; N, 8.91\%; Found: C, 64.90; H, 5.75; N, 8.86\%.

\section{1-Methyl-2-[1-toluene-4-sulfonyl)-ethyl]-1H-benzimidazole (3h)}

M.P. $\left({ }^{\circ} \mathrm{C}\right): 182 ; \operatorname{IR}(\mathrm{KBr}):{ }^{1} \mathrm{H}$ NMR $\left(\mathrm{CDCl}_{3}\right): \delta 1.70\left(\mathrm{~d}, J=7.18 \mathrm{~Hz}, 3 \mathrm{H},-\mathrm{CH}-\mathrm{CH}_{3}\right), 3.85$ (s, $\left.3 \mathrm{H},-\mathrm{NCH}_{3}\right), 5.10\left(\mathrm{q}, J=7.0 \mathrm{~Hz}, 1 \mathrm{H},-\mathrm{CH}-\mathrm{CH}_{3}\right), 7.2-7.87$ (m, 9H, Ar-H); $\mathrm{M}^{+}+1: 301$; Anal. Calcd. for $\left(\mathrm{C}_{16} \mathrm{H}_{16} \mathrm{~N}_{2} \mathrm{O}_{2} \mathrm{~S}\right)$ requires: C, 63.98; H, 5.37; N, 9.33\%; Found: C, 63.95; H, 5.33; $\mathrm{N}, 9.29 \%$.

\section{1-Methyl-4-phenylmethanesulfonyl-benzene (3i)}

M.P. $\left({ }^{\circ} \mathrm{C}\right): 190-201$; IR $(\mathrm{KBr}) \mathrm{cm}^{-1}: 1312\left(\mathbf{S O}_{2}\right) ;{ }^{1} \mathrm{H}$ NMR $\left(\mathrm{CDCl}_{3}\right): \delta 2.38\left(\mathrm{~s}, 3 \mathrm{H},-\mathrm{CH}_{3}\right)$, $4.61\left(\mathrm{~s}, 2 \mathrm{H},-\mathrm{CH}_{2}\right), 7.11-7.57\left(\mathrm{~m}, 9 \mathrm{H}\right.$, aromatic protons); $\mathrm{M}^{+}+1: 247$.

\section{Results and Discussion}

In our initial attempts, triethanol amine was used in the place of triethyl benzylammonium chloride (TEBAC) $)^{21}$, which acts as phase transfer catalyst (PTC), in acetonitrile as solvent, used for the synthesis of $1 H-2$-( $p$-tolylsulfonyl methyl)benzimidazole. To verify the existence of such an auxiliary effect, we have investigated the use of triethanol amine as reaction medium which itself gives relief from using phase transfer catalyst as well as from using additional solvent (Table 1$)$. It was found that the condensation of 2 - $(\alpha$-chloromethyl) benzimidazole 1a (i.e. 1, $\mathrm{R}=\mathrm{H}$ ) with $p$-tolylsulphinate sodium salt 2a (i.e. $2, \mathrm{Ar}=\mathrm{C}_{6} \mathrm{H}_{4}$ $\left.\mathrm{CH}_{3}(p)\right)$ in triethanol amine at room temperature yielded a neat product on simple workup. The product has been characterized as $1 H$-2-( $p$-tolylsulfonyl methyl)benzimidazole 3a (i.e. $3, \mathrm{R}=\mathrm{H}, \mathrm{Ar}=\mathrm{C}_{6} \mathrm{H}_{4}-\mathrm{CH}_{3}(p)$ ), based on spectral and analytical data and also it was found to be identical with the product reported under phase transfer catalytic conditions ${ }^{21}$. 


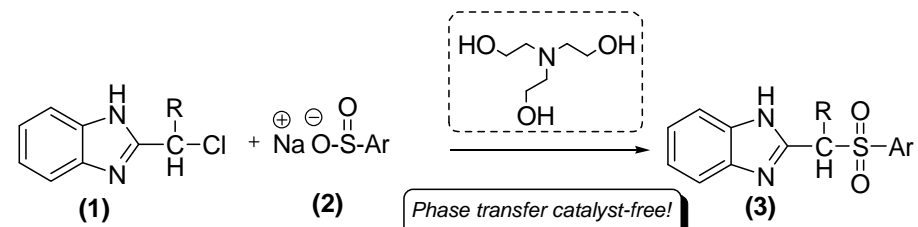

Scheme 1

The plausible mechanism: (for formation of 3 )
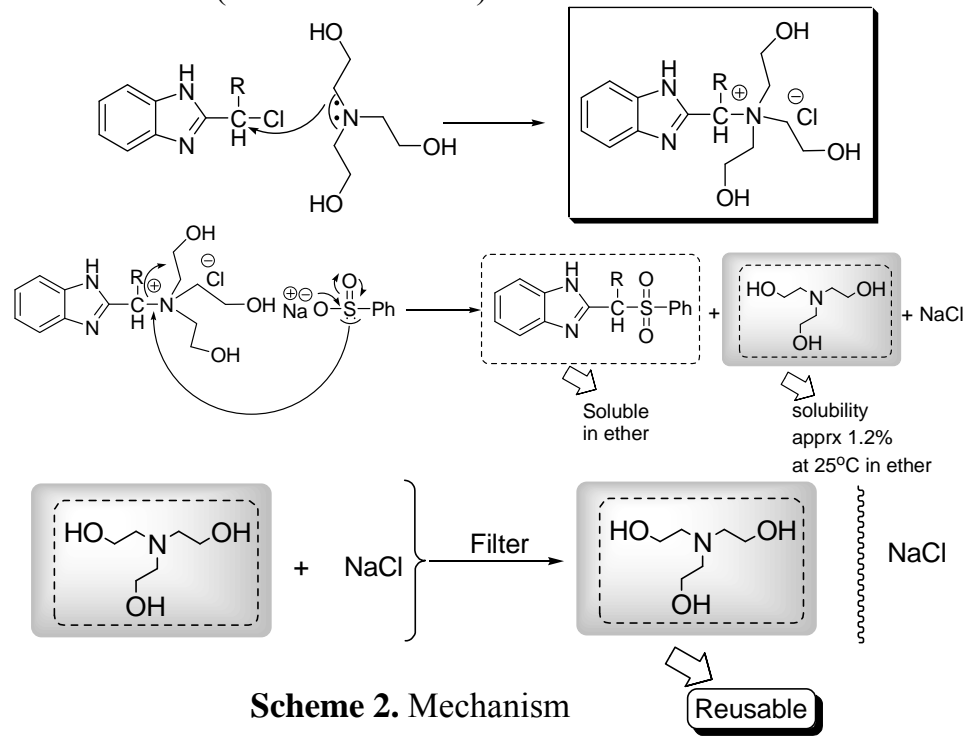

Scheme 2. Mechanism

Table 1. (In general)

\begin{tabular}{ccccc}
\hline Entry & Solvent & Catalyst & Reaction, time h & Yield \% \\
\hline 1 & $\mathrm{CH}_{3} \mathrm{CN}$ & TEBAC & $3-4$ & $70-85$ \\
2 & $\mathrm{CH}_{3} \mathrm{CN}$ & TEOA & $3-3.5$ & $75-85$ \\
3 & TEOA & - & $1.5-2.0$ & $80-89$ \\
\hline
\end{tabular}

The above reaction has been found to be a general one and has been extended to other 1a \& 2a i.e. with various halo compounds and sulfinate derivatives, which resulted in the formation of different sulphones (Table 2).

Table 2. Triethanolamine: A resourceful, reusable, reaction medium for phase transfer catalyst - free synthesis of 1-(arylsulfonyl) aryl/heteryl methanes

\begin{tabular}{ccccc}
\hline Entry & Reagent & Product (3) & Time, h & Yield, \% \\
\hline 1a & $\mathbf{2 a}$ & & & \\
& & &
\end{tabular}

1a

2b

(3a)

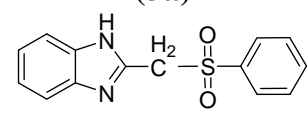

1.5

81

(3b) 


1d

\section{General reaction}

In order to examine the scope of this method in preparing different sulphone derivatives, we have examined the reactions of benzyl chlorides and phenacyl bromides with sodium benzene sulfinates under the above mentioned conditions. As shown in table, moderate to good yields of the title compounds were obtained.<smiles>CC(C)CCO</smiles>

(2)

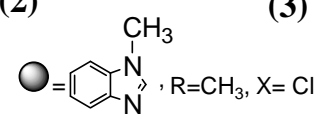

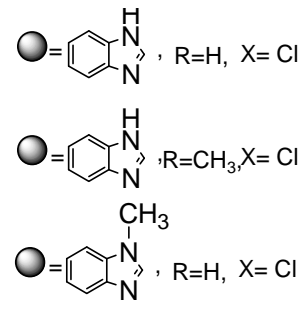

$\mathrm{O}=\mathrm{Ph}, \mathrm{R}=\mathrm{H}, \mathrm{X}=\mathrm{Cl}$

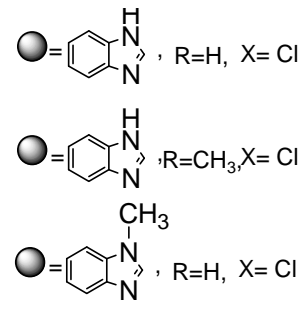

$\mathrm{O}=\mathrm{Ph}-\stackrel{\mathrm{O}}{\mathrm{C}}-\mathrm{R}=\mathrm{H}, \mathrm{X}=\mathrm{Br}$

\section{Scheme 3}

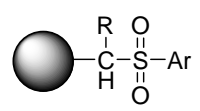

(3) 


\section{Recyclability of triethanolamine}

After carrying out the reaction, the mixture was filtered and the filtrate extracted with diethyl ether (note: the solubility of Triethanolamine in diethyl ether is approx $1.2-1.4 \%$ at $\left.25^{\circ} \mathrm{C}\right)^{22}$. Triethanolamine was separated and washed successively with $\mathrm{Et}_{2} \mathrm{O}(2 \times 5 \mathrm{~mL})$ and hexane $(5 \mathrm{~mL})$ in order to remove adsorbed organic substrates. It can be reused directly without further purification.

\section{Conclusion}

We have presented herein an efficient method for the synthesis of title compounds using triethanolamine as the eco-friendly solvent in good to excellent yields. Moreover, this method is advantageous than the previously reported method by us and Triethanolamine could be recovered and recycled for five consecutive trials without significant loss in activity. The triethanolamine itself and its products of degradation are not toxic ${ }^{22}$.

\section{Acknowledgment}

The authors are highly indebted to Council of Scientific and Industrial Research (CSIR), Govt. of India, New Delhi, for financial support (Senior Research Fellowship-SRF to Mr. K. Srinivas). They are also thankful to the authorities of Jawaharlal Nehru Technological University Hyderabad for providing laboratory facilities.

\section{References}

1. a) Sturino C F, Neill G, Lachance N, Boyd M, Bertgelette C, Labelle M, Li L, Roy B, Scheigetz J, Tsou N, Aubin Y, Bateman K.P, Chauret N, Day S H, Levesque J F, Seto C, Silva J H, Trimble L A, Carriere M C, Denis D, Greig G, Kargman S, Lamontagne S, Mathieu M C, Sawyer N, Slipetz D, Abraham W M, Jones T, McAuliffe M, Piechuta H, Nicoll-Griffith D A, Wang Z, Zamboni R, Young R.N and Metters K M, J Med Chem., 2007, 50(4), 794-806; DOI:10.1021/jm0603668; b) Trost B M, Shen H C and Surivet J P, J Am Chem Soc., 2004, 126(39), 12565-12579; DOI: 10.1021/ja048084p; c) Adams C M, Ghosh I and Kishi Y, Org Lett., 2004, 6(25), 4723-4726; DOI:10.1021/ol048059o; d) Artico M, Silvestri R, Pagnozzi E, Bruno B, Novellino E, Greco G, Massa S, Ettorre A, Loi A G, Scintu F and La Colla P, J Med Chem., 2000, 43(9), 1886-1891; DOI:10.1021/jm9901125; e) Michaely W J, Krattz G W, US. Patent 4, 780, 127, 1988; Chem Abstr., 1989, 111, 129017; f) Kochi T, Noda S, Yoshimura K and Nozaki K, J Am Chem Soc., 2007, 129(29), 8948-8949; DOI:10.1021/ja0725504; g) Wei X L, Wang Y Z, Long S M, Bobeczko C and Epstein A J, J Am Chem Soc., 1996, 118(11), 2545-2555; DOI:10.1021/ja952277i.

2. a) Neamati N, Mazumder A, Zhao H, Sunder S, Jr Burke T R, Schultz R J and Pommier Y, Antimicrob Agents Chemother., 1997, 41(2), 385-393; b) Artico M, Silvestri R, Massa S, Loi A.G, Corrias S, Piras G and La Colla P, J Med Chem., 1996, 39(2), 522-530; DOI:10.1021/jm950568w: c) McMahon J B, Gulakowsky R J, Weislow O S, Schultz R J, Narayanan V L, Clanton D J, Pedemonte R, Wassmudt F W, Buckheit R W, Decker W D, White E L, Bader J P and Boyd M.R, Antimicrob Agents Chemother., 1993, 37(4), 754-760; DOI:10.1128/AAC.37.4.754.

3. Williams T M, Ciccarone T M, Mac Tough S C, Rooney C S, Balani S K, Condra J H, Emini E A, Goldman M E, Greenlee W J, Kauffman L R, O’Brien J A, Saradana V V, Schleif W A, Theohardes A D and Anderson P S, J Med Chem., 1993, 36(9), 12911294; DOI:10.1021/jm00061a022. 
4. Repichet S, Le Roux C, Hernandez P, Dubac J and Desmurs J R, J Org Chem., 1999, 64(17), 6479-6482; DOI:10.1021/jo9902603.

5. a) Kar A, Sayyed I A, Lo W F, Kaiser H M, Beller M and Tse M F, Org Lett., 2007, 9(17), 3405-3408; DOI:10.1021/ol071396n; b) Kuwano R, Kondo Y and Shirahama T, Org Lett., 2005, 7(7), 2973-2975; DOI:10.1021/o10509787; c) Cacchi S, Fabrizi G, Goggiamani A and Parisi L M, Org Lett., 2002, 4(26), 4719-2721.

6. a) Jensen F R and Goldman G, In Friedel Crafts and Related Reactions; Olah G A, Ed., Wiely-Interscience: Newyork, 1964; III, 1319; b) Taylor R, In Comprehensive Chemical Kinetics; Banford C H and Tipper C F H, Eds., Elsevier- New York, 1972, 77; c) Alexander M V, Khandekar A C and Samant S D, J Mol Catal A: Chem., 2004, 223(1-2), 75-83; DOI:10.1016/j.molcata.2003.10.066.

7. a) Ono M, Nakamura Y, Sato S and Itoh I, Chem Lett., 1988, 17(3) 395-398; DOI:10.1246/cl.1988.395; b) Effenberger F and Huthmacher K, Chem Ber., 1976, 109(6), 2315-2326; DOI:10.1002/cber.19761090636.

8. a) Emmerson D P G, Hems W P and Davis B G, Org Lett., 2006, 8(2), 207-210; DOI:10.1021/o10525031; b) Li Z, Upadhyay V, DeCamp A E, DiMichele L and Reidor P J, Synthesis, 1999, 1453-1458; c) Lu G, Li X, Zhou Z, Chan W.L and Chan A S C, Tetrahedron: Asymmetry, 2001, 12(15), 2147-2152.

9. a) Tanyeli C and Suenbuel M, Tetrahedron: Asymmetry, 2005, 16(11), 2039-2043; DOI:10.1016/j.tetasy.2005.04.019; b) Jimeno C, Pasto M, Riera A and Pericas M.A, $J$ Org Chem., 2003, 68(8), 3130-3138; DOI:10.1021/jo0340071; c) Nevalainen M and Nevalainen V, Tetrahedron: Asymmetry, 2001, 12(12), 1771-1777; DOI:10.1016/S0957-4166(01)00295-6; d) Soai K, Hatanaka T and Miyazawa T, $J$ Chem Soc Chem Commn., 1992, 1097; DOI:10.1039/C39920001097; e) Brandt P, Hedberg C, Lawonn K, Pinho P and Andersson P G, Chem Eur J., 1999, 5, 1692-1699; DOI:10.1002/(SICI)1521-3765(19990604)5:6<1692::AID-CHEM1692>3.0.CO;2-M.

10. a) Wu X, Li X, McConville M, Saidi O and Xiao J, J Mol Catal A, 2006, 247(1-2), 153-158; DOI:10.1016/j.molcata.2005.11.040; b) Patti A and Pedotti S, Tetrahedron: Asymmetry, 2003, 14(5), 597-602; DOI:10.1016/S0957-4166(02)00835-2.

11. You J S, Gau H M and Choi M C K, J Chem Soc Chem Commun., 2000, 1963.

12. Palomo C, Oiarbide M and Laso A, Angew Chem Int Ed., 2005, 44(25), 3881-3884; DOI: 10.1002/anie.200463075.

13. Klapars A, Antilla J C, Huang X and Buchwald S L, J Am Chem Soc., 2001, 123(31), 7727-7729; DOI:10.1021/ja016226z.

14. Kwong F Y, Klapars A and Buchwald S L, Org Lett., 2002, 4(4), 581-584; DOI:10.1021/ol0171867.

15. Gonzalez-Bobes F and Fu G C, J Am Chem Soc., 2006, 128(16), 5360-5361; DOI:10.1021/ja0613761.

16. Mukhopadhyay S, Rothenberg G, Joshi A, Baidossi M and Sasson Y, Adv Synth Catal., 2002, 344(3-4), 348-354.

17. Dubey P K, Prasada Reddy P V V and Srinivas K, Synth Commun., 2007, 37(10), 1675-1681; DOI:10.1080/00397910701265556.

18. Dubey P K, Prasada Reddy P V V and Srinivas K, Lett Org Chem., 2007, 4, 445.

19. Dubey P K, Prasada Reddy P V V and Srinivas K, Arkivoc, 2007, xv, 192-198.

20. Dubey P K, Prasada Reddy P V V and Srinivas K, Synth Commun., 2008, 38, 619-625.

21. Dubey P.K, Prasada Reddy P.V.V and Srinivas K, Indian J Chem Sec-B, 2007, 46, 488.

22. Material Safety Data Sheet, Triethanolamine, Science Lab. Com., CAS\#:102-71-6, http://www.sciencelab.com /msds.php?msdsId=9927306

23. Hong J L and Wang L, Eur J Org Chem., 2006, 5099-5102. 